\title{
Uso de Parâmetros Ósseos, Plasmáticos e Fecais na Determinação da Deficiência de Fósforo em Bovinos
}

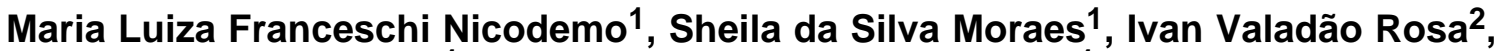 \\ Manuel Cláudio Motta Macedo ${ }^{1}$, Luiz Roberto Lopes de S. Thiago', Cláudio Ribeiro dos Anjos ${ }^{3}$
}

RESUMO - Quinze novilhos Nelore foram distribuídos em três tratamentos, consistindo de dieta basal capaz de proporcionar ganhos da ordem de $600 \mathrm{~g} /$ dia, suplementada ( 15 ou $11 \mathrm{~g} \mathrm{P} /$ dia) ou não (5 g P/dia) com fosfato monoamônico, para determinar as alterações provocadas por deficiência de $\mathrm{P}$ em parâmetros ósseos, sangüíneos e fecais. Biópsias de costela foram obtidas aos 60 e 250 dias de experimentação. Amostras de plasma foram coletadas quinzenalmente e amostras de fezes retais, analisadas em sete ocasiões, durante os 250 dias do experimento. Coleta total (diária) de fezes foi feita em dois períodos, aos 60 e 243 dias, durante sete dias. Aos 60 dias, $\mathrm{P}$ expresso em relação ao osso fresco, osso seco e desengordurado e ao volume de osso discriminaram entre os tratamentos. Aos 250 dias, a espessura da camada cortical e as concentrações de cinzas e fósforo foram reduzidas na deficiência de P. Observou-se queda na concentração de fósforo inorgânico plasmático (Pi), já aos 12 dias de experimentação, apresentando valores em torno de 3,0 mg \% na época em que ganho de peso foi afetado ( 82 dias). Foi observada elevação do Ca e da fosfatase alcalina na deficiência de P, sem alterações na concentração de hidroxiprolina do plasma. As concentrações de P nas fezes aumentaram com a concentração do elemento na dieta, mas a utilização de amostras coletadas do reto não propiciou detecção precoce de deficiência de P. A definição de níveis críticos para a determinação de deficiência apresentou problemas.

Palavras-chave: bovinos, deficiência, fósforo, níveis críticos, osso, plasma

\section{Use of Bone, Plasma and Feces in the Assessment of $\mathbf{P}$ Status in Cattle}

\begin{abstract}
Fifteen Nellore steers were allotted to three treatments, consisting of a basal diet, which will be able to provide gains of about $600 \mathrm{~g} /$ day, supplemented ( 15 or $11 \mathrm{~g}$ P/day) or not ( $5 \mathrm{~g}$ P/day) with monoammonium phosphate in order to evaluate the alterations in bone, plasma and fecal measurements due to P deficiency. Rib biopsies were taken at 60 and 250 days of trial, plasma samples were taken every 15 days, and fecal grab samples were analyzed in seven occasions along the trial. Total daily fecal collection was performed in two occasions, at 60 and 243 days of the trial, for seven days. At day 60, P expressed as fresh bone, dried defatted bone and bone volume were able to detect treatment differences. At day 250, cortical thickness, $\mathrm{P}$ and ash concentrations were reduced in P deficiency. Plasma inorganic P (Pi) was lower at 12 days of trial, with values close to 3,0 $\mathrm{mg} \%$ at the time where daily weight gain was affected (day 82). Calcium and total alkaline phosphatase increased in P deficiency, without changes on the plasma hidroxyproline concentration. Fecal P concentrations increased as the dietary P concentrations increased, but grab samples did not allow early detection of $\mathrm{P}$ deficiency. The definition of critical P levels for the determination of deficiency showed problems.
\end{abstract}

Key Words: cattle, deficiency, phosphorus, critical levels, bone, plasma

\section{Introdução}

Sinais clínicos da deficiência crônica de fósforo, como baixo ganho de peso, consumo reduzido, depravação de apetite e falha reprodutiva (UNDERWOOD, 1981), podem levar certo tempo para se estabelecerem. A identificação de deficiências marginais, nas quais existem perdas produtivas sem sintomas clínicos definidos, exige o uso de análises bioquímicas. A determinação da deficiência de um elemento mineral pode se dar por intermédio de monitoração dos níveis do mesmo nos tecidos ou de comparação das estimativas de ingestão do elemento com os requisitos nutricionais. Os tecidos mais utilizados na avaliação da condição de fósforo são sangue e osso. A concentração de fósforo inorgânico no plasma ou soro é muito usada, já que o teor de fósforo inorgânico cai rapidamente, quando a dieta é inadequada. É importante o estabelecimento dos níveis de $\mathrm{P}$ no sangue no momento em que a deficiência de $\mathrm{P}$ começa a afetar o consumo de alimento, produzindo prejuízos econômicos. O osso é considerado o tecido mais indicado

\footnotetext{
1 Pesquisador da Embrapa Gado de Corte. Caixa Postal 154, CEP 79002-070, Campo Grande, MS. E.mail: sgcnpgc@cnpgc.embrapa.br

${ }^{2}$ Médico-veterinário. Pesquisador Aposentado da Embrapa Gado de Leite.

${ }^{3}$ Assistente de Pesquisa da Embrapa Agropecuária Oeste.
} 
para a avaliação das reservas de $\mathrm{P}$ do animal, por conter $80 \%$ do fósforo, que pode ser utilizado, quando há deficiência nutricional. Os valores utilizados na avaliação dos índices ósseos muitas vezes não são hábeis na detecção de deficiências, em virtude do grau de esgotamento das reservas ósseas necessário para aparecerem diferenças significativas e, talvez, da não-disponibilidade de parte do mineral depositado. Procurou-se, assim, avaliar o tempo necessário para o aparecimento de alterações significativas em parâmetros ósseos, observando-se também a sensibilidade dos mesmos. Por outro lado, a metodologia recomendada para estimar a quantidade de P ingerida pelo animal envolve a utilização de radioisótopos para identificar a fração de origem endógena (LITTLE et al., 1977). A possibilidade de haver algum parâmetro alternativo, de precisão aceitável, levou alguns pesquisadores a relacionar $\mathrm{P}$ fecal ao $\mathrm{P}$ na dieta. $\mathrm{O} \mathrm{P}$ ingerido tem estreita relação com $\mathrm{P}$ salivar, que é a principal rota de excreção de $\mathrm{P}$ nas fezes (SCOTT et al., 1985; CHALLA et al., 1989). Níveis de P em amostras de fezes coletadas do reto inferiores a $0,12 \%$ na matéria seca são considerados indicadores de deficiência por pesquisadores australianos (RESOURCE CONSULTING SERVICES, 1986) e têm auxiliado no manejo da suplementação de P. Os objetivos deste trabalho foram: a) avaliar a utilização de biópsia de osso na indicação das reservas de P de novilhos Nelore em crescimento; b) determinar as relações entre o P plasmático e a deficiência subclínica de $\mathrm{P}$, avaliando também as alterações nas concentrações plasmáticas de cálcio, fosfatase alcalina e hidroxiprolina; e c) determinar a viabilidade da utilização da concentração de $\mathrm{P}$ nas fezes para a indicação da deficiência de P.

\section{Material e Métodos}

Quinze novilhos Nelore, de cerca de $215 \mathrm{~kg}$, foram distribuídos em três tratamentos, consistindo de dieta basal capaz de proporcionar ganhos de peso da ordem de $600 \mathrm{~g} /$ dia, suplementada (tratamento $1=$ 15 ou tratamento $2=11 \mathrm{~g} \mathrm{P} /$ dia) ou não (tratamento $3=5 \mathrm{~g}$ P/dia) com fosfato monoamônico. Os animais foram pesados a cada 15 dias e o consumo diário individual de matéria seca foi monitorado. Foram feitos exames clínicos mensais nos animais, consistindo de observação do estado geral, exame de pele e pêlo, exame da cavidade bucal, exame de mucosas, temperatura retal, aspecto e consistência das fezes, auscultação dos ritmos cardíaco e respiratório. Amos- tras de costela foram obtidas de todos os novilhos aos 60 e 250 dias de experimento, utilizando a técnica desenvolvida por LITTLE (1972). Nas amostras de osso, foram analisadas as concentrações de P, Ca e cinza (FICK et al., 1980), a gravidade específica e espessura da camada cortical (LITTLE, 1984). Amostras de sangue foram coletadas da jugular, quinzenalmente, em frascos contendo heparina (para análise de fosfatase alcalina) ou EDTA (outras análises). O plasma foi centrifugado (3.000 rpm, 15 minutos) e separado logo após a coleta, sendo mantido a $-20^{\circ} \mathrm{C}$ até a análise. No plasma, foram analisados os teores cálcio e fósforo inorgânico (FICK et al., 1980), fosfatase alcalina (total) (Labtest Diagnóstica S.A., Belo Horizonte, Brasil), hidroxiprolina (JAESCHKE, 1975) e porcentagem de eritrócitos. Amostras de fezes foram coletadas do reto, quinzenalmente, durante os 250 dias do experimento. Apenas sete dessas coleções puderam ser utilizadas, devido a problemas com a identificação das mesmas. Coleta total (diária) de fezes foi feita em dois períodos, aos $60 \mathrm{e}$ 243 dias de experimentação, durante sete dias. Amostras do alimento oferecido e rejeitado foram coletadas diariamente, obtendo-se um pool semanal para cada animal. Nas fezes e no alimento, foram analisadas as concentrações de P (FICK et al., 1980), matéria seca e matéria orgânica (HARRIS, 1970). As análises estatísticas foram realizadas por intermédio de regressão e análise de variância (ANOVA), conforme procedimento do aplicativo SAS (SAS, 1985).

\section{Resultados e Discussão}

O ganho de peso dos animais reduziu-se $(\mathrm{P}<0,01)$ significativamente aos 71 dias de deficiência de P. Aos 60 dias de experimento, foi feita a primeira biópsia de osso. Nessa ocasião, observou-se queda nas concentrações de P no osso, em função dos menores níveis de $P$ na dieta, quando essas concentrações foram expressas em relação ao osso fresco, osso seco e desengordurado e ao volume de osso (Tabela 1). Outros autores encontraram menor sensibilidade na medida expressa como osso seco e desengordurado (LITTLE e McMENIMAN, 1975; READ et al., 1986), devido ao fato de a redução da mineralização do osteóide na deficiência de P estar acompanhada por aumento no teor de água e lípides (ECKLES et al., 1932; LITTLE, 1972; e BELONJE, 1978).

A razão $\mathrm{Ca} / \mathrm{P}$ (Tabela 2) também foi afetada por nível de $\mathrm{P}$ na dieta aos 60 dias de experimento. $\mathrm{O}$ cristal de apatita pode ser descrito pela fórmula geral: 
842 Rev. bras. zootec.

Tabela 1 - Regressão linear da concentração de $P$ no osso fresco (POF) e osso seco e desengordurado (POSD), nas cinzas (PC) e no volume de osso (PV), aos 60 e 250 dias de experimentação, em função do nível $(\mathrm{N})$ de $\mathrm{P}$ na dieta

Table 1 - Linear regressions of concentrations of $P$ in fresh bone (POF), dried defatted bone (POSD), ashes $(P C)$ and bone volume (PV) at 60 and 250 experimental days on the $P$ levels $(N)$ in the diet

\begin{tabular}{lclcc}
\hline Item & Dias & $\begin{array}{l}\text { Regressão } \\
\text { Regression }\end{array}$ & $\begin{array}{c}\text { Significância do efeito } \\
\text { Significance of the effect }\end{array}$ & $\mathrm{r}^{2}$ \\
\hline POF, \% & 60 & POF $=7,66+0,074 \mathrm{~N}$ & $*$ & 0,34 \\
& 250 & $\mathrm{POF}=7,37+0,103 \mathrm{~N}$ & $* * *$ & 0,56 \\
POSD, \% & 60 & $\mathrm{POSD}=10,08+0,040 \mathrm{~N}$ & $*$ & 0,34 \\
& 250 & $\mathrm{POSD}=10,00+0,088 \mathrm{~N}$ & $* * *$ & 0,68 \\
PC, $\%$ & 60 & $\mathrm{PC}=17,8$ & $* * *$ & 0,55 \\
& 250 & $\mathrm{PC}=17,2+0,073 \mathrm{~N}$ & $*$ & 0,35 \\
PV, mg/cc & 60 & $\mathrm{PV}=126+2,01 \mathrm{~N}$ & $* *$ & 0,48 \\
& 250 & $\mathrm{PV}=121+2,47 \mathrm{~N}$ & &
\end{tabular}

Níveis de significância (ANOVA): ${ }^{*} \mathrm{P}<0,05 ;{ }^{* *} \mathrm{P}<0,01 ;{ }^{* *} \mathrm{P}<0,001$.

Levels of significance (ANOVA): ${ }^{*} P<.05 ;{ }^{* *} P<.01 ;{ }^{* * *} P<.001$.

Tabela 2 - Regressão linear das concentrações de cinzas no osso fresco (COF) e no osso seco e desengordurado (COSD), Ca nas cinzas e razão Ca/P, aos 60 e 250 dias de experimentação, em função do nível $(\mathrm{N})$ de $\mathrm{P}$ na dieta

Table 2 - Linear regressions of concentrations of ash in fresh bone (COF) and defatted dried bone (COSD), $\mathrm{Ca}$ in the ashes and $\mathrm{Ca} / \mathrm{P}$ ratio at 60 and 250 experimental days as the $P$ levels $(N)$ in the diet

\begin{tabular}{|c|c|c|c|c|}
\hline Item & $\begin{array}{l}\text { Dias } \\
\text { Days }\end{array}$ & $\begin{array}{l}\text { Regressão } \\
\text { Regression }\end{array}$ & $\begin{array}{l}\text { Significância do efeito } \\
\text { Significance of the effect }\end{array}$ & $\mathrm{r}^{2}$ \\
\hline \multirow[t]{2}{*}{$\mathrm{COF}(\%)$} & 60 & $\mathrm{COF}=43,3$ & & - \\
\hline & 250 & $\mathrm{COF}=42,9+0,383 \mathrm{~N}$ & $* *$ & 0,40 \\
\hline \multirow[t]{2}{*}{$\operatorname{COSD}(\%)$} & 60 & $\operatorname{COSD}=60,6$ & & - \\
\hline & 250 & $\operatorname{COSD}=58,2+0,241 \mathrm{~N}$ & $* *$ & 0,46 \\
\hline \multirow[t]{2}{*}{$\mathrm{Ca}(\%)$} & 60 & $\mathrm{Ca}=43,4$ & & - \\
\hline & 250 & $\mathrm{Ca}=42,8$ & & - \\
\hline \multirow{2}{*}{$\mathrm{Ca} / \mathrm{P}$} & 60 & $\mathrm{Ca} / \mathrm{P}=2,44-0,014 \mathrm{~N}$ & $* *$ & 0,45 \\
\hline & 250 & $\mathrm{Ca} / \mathrm{P}=2,49-0,013 \mathrm{~N}$ & $*$ & 0,37 \\
\hline
\end{tabular}

Níveis de significância (ANOVA): * $\mathrm{P}<0,05 ;{ }^{* *} \mathrm{P}<0,01$.

Levels of significance $\left(\right.$ ANOVA): ${ }^{\star} P<.05 ;{ }^{*} P<.01$.

$\mathrm{Ca}{ }_{8,3}\left(\mathrm{PO}_{4}\right)_{4,3}\left(\mathrm{CO}_{3}\right)_{\mathrm{X}}\left(\mathrm{HPO}_{4}\right)_{\mathrm{Y}}(\mathrm{OH})_{0,3},(\mathrm{X}+\mathrm{Y})=$ 1,7. Em contraste com o nível de $\mathrm{Ca}$, que permanece constante no cristal, os íons fosfato podem ser substituídos por carbonatos (LEGROS et al., 1987). Aumento na razão $\mathrm{Ca} / \mathrm{P}$, na deficiência de $\mathrm{P}$, também foi descrito por SHIRAZI-BEECHEY et al. (1991) e WAN ZAHARI et al. (1990), em carneiros.

Ambas a composição química e a estrutura do osso são afetadas na deficiência de P. Existem indicações de redução da formação de osso em ovinos (CORLETT e CARE, 1988; SCOTT et al., 1994) e da mineralização do osso em ovinos (SHIRAZI-BEECHEY et al., 1991; TERNOUTH et al., 1993; e SCOTT et al., 1997) e bovinos (SHUPE et al., 1988). A deficiência de P também tem sido associada ao balanço negativo de P (TERNOUTH e SEVILLA, 1990; SHIRAZI-BEECHEY et al., 1991; e BORTOLUSSI et al., 1996) e aumento na excreção de piridinolina e deoxipiridinolina (SCOTT et al., 1997), em ruminantes, indicando reabsorção do osso e/ou perda de $\mathrm{P}$ dos tecidos moles.

Diminuição na retenção de Ca tem sido frequientemente relatada na deficiência de $\mathrm{P}$ em ruminantes (CHALLA et al., 1989), dando respaldo à tese de redução na formação de osso; assim, não há surpresa ao se constatar que, após 250 dias, a maioria dos parâmetros avaliados no osso (concentração de fósforo [Tabela 1] e de cinzas [Tabela 2], e espessura da cortical [Tabela 3]) foi significativamente reduzida na deficiência de fósforo. As equações de regressão foram lineares para todos os parâmetros ósseos medidos, confirmando observações de que os requisitos para máxima mineralização óssea são maiores que aqueles para máxima produção (WISE et al., 1958; UNDERWOOD, 1966).

Os níveis de $\mathrm{P}$ utilizados na definição de deficiência são empíricos, pois baseiam-se, de modo geral, na comparação entre os valores de animais sujeitos a dietas deficientes e os valores encontrados em animais suplementados ou sem sintomas clínicos de 
NICODEMO et al.

Tabela 3 - Regressão linear da densidade (DENS) e espessura da camada cortical (CORT) no osso, aos 60 e 250 dias de experimentação, em função do nível (N) de P na dieta

Table 3 - Linear regressions relating bone density (DENS) and cortical thickness (CORT) at 60 and 250 experimental days on the $P$ levels $(N)$ in the diet

\begin{tabular}{lclcc}
\hline Item & $\begin{array}{c}\text { Dias } \\
\text { Days }\end{array}$ & $\begin{array}{c}\text { Regressão } \\
\text { Regression }\end{array}$ & $\begin{array}{c}\text { Significância do efeito } \\
\text { Significance of the effect }\end{array}$ & $\mathrm{r}^{2}$ \\
\hline CORT, cm & 60 & CORT $=2,36$ & & - \\
& 250 & CORT $=1,88+0,120 \mathrm{~N}$ & $* *$ & 0,52 \\
DENS & 60 & DENS $=1,66$ & & - \\
& 250 & DENS $=1,65$ & & - \\
\hline
\end{tabular}

Níveis de significância (ANOVA): **, $\mathrm{P}<0,01$

Levels of significance (ANOVA): **, $P<.01$.

deficiência de P. Em razão de existirem diferenças quanto à idade, ao estádio físiológico e às condições de manejo e alimentação entre os animais, divergências são comuns, uma vez que todos esses fatores, às vezes combinados, podem afetar composição e estrutura do osso (MELLO JR. et al., 1978; READ et al., 1986; LAWRENCE e FOWLER, 1997; NICODEMO, 1997; e SCOTT et al., 1997).

Aos 250 dias, quando se comparam as concentrações medidas com os níveis críticos sugeridos, nenhum tratamento estaria inadequado, se consideradas a espessura da cortical (valor de referência: LITTLE, 1984), a densidade (valores de referência: SHUPE et al., 1988; LITTLE, 1972; e READ et al., 1986) e a concentração de $P$ expressa por volume (valor de referência: LITTLE e SHAW, 1979). Contudo, se consideradas as concentrações de cinzas ou de $\mathrm{P}$ no osso seco e desengordurado, os tratamentos poderiam ser considerados deficientes (valores de referência: LITTLE, 1972; McDOWELL, 1985). Essas observações encontram respaldo nas críticas de HOEY et al. (1982) e WADSWORTH (1990), os quais relatam que muitos fatores, além do aporte de $\mathrm{P}$, influenciam os valores normais do elemento no osso, limitando seu valor diagnóstico. É possível que o uso de padrões locais reduza esse problema (WADSWORTH et al., 1990). É improvável que um único critério possa ser usado; a combinação de vários parâmetros, inclusive bioquímicos, parece ser o caminho mais lógico na identificação de animais com deficiência subclínica de P.

$\mathrm{Na}$ deficiência simples de $\mathrm{P}$, o plasma tem se mostrado bom indicador da condição animal. Os animais do tratamento 3 apresentaram níveis inferiores de $\mathrm{P}$ no plasma já à primeira coleta, aos 12 dias de experimento $(8,12 ; 5,73$; e $4,33 \mathrm{mg} \%$ para os tratamentos 1,2 e 3 , respectivamente). Os teores plasmáticos de $\mathrm{P}$ inorgânico $(\mathrm{Pi})$ no tratamento 3 foram claramente inferiores aos observados no trata- mento 1 (Tabela 4), refletindo a ingestão de dieta pobre em $\mathrm{P}$, já que existe relação linear direta entre ingestão de $\mathrm{P}$ e concentração do elemento no plasma (SCOTT et al., 1985).

A queda na ingestão de alimento $(\mathrm{P}<0,05)$ foi observada a partir de 42 dias de experimento; o ganho de peso mostrou-se reduzido $(\mathrm{P}<0,01)$ aos 71 dias. Os valores observados nesta fase são críticos para a determinação da deficiência subclínica de P. A queda dos níveis plasmáticos de $\mathrm{Pi}$ no tratamento 3 deu-se nesta fase inicial, permanecendo ao redor de 3,0 $\mathrm{mg} \%$, a partir de 82 dias de experimento $(\mathrm{P}>0,05)$.

Os níveis de Pi plasmáticos observados em novilhos deficientes em P (3,0 mg\%) estão de acordo com os valores críticos sugeridos por GARTNER et al. (1980) e WINTER (1988), portanto, inferiores aos 4,5 mg\% sugeridos por McDOWELL (1985). Uma explicação para o equilíbrio nos níveis de $\mathrm{Pi}$ plasmáticos nos novilhos deficientes, embora em patamares inferiores, é a capacidade de adaptação de ruminantes à deficiência de $\mathrm{P}$, por intermédio de mecanismos como o aumento da eficiência de absorção do P no intestino (SHIRAZI-BEECHEY et al., 1991; COATES e TERNOUTH, 1992).

A redução do Pi plasmático no tratamento 1 foi inesperada, já que esses animais recebiam dieta adequada. Os níveis de fósforo são normalmente mais elevados em animais jovens e, devido à manutenção dos valores de Pi no tratamento 2, durante todo o período experimental, pode ser descartado possível efeito da idade. Uma possibilidade seria de que a elevada taxa de crescimento desses animais ocasionaria a redução dos níveis no plasma de maneira análoga à redução da concentração de $\mathrm{P}$ no osso observada em animais com altas taxas de crescimento (BROMMAGE et al., 1992; NICODEMO, 1997). Com o rápido crescimento dos tecidos, maior quantidade de $\mathrm{P}$ estaria mobilizada para formação e metabolismo de ossos e demais tecidos. 
844 Rev. bras. zootec.

Tabela 4 - Regressão linear relacionando $\mathrm{P}$ inorgânico $(\mathrm{Pi}$, $\mathrm{mg} \%$ ) e Ca plasmático ( $\mathrm{mg} \%$ ) em função do tempo de experimentação (dias, D) nos tratamentos

Table 4 - Linear regressions relating inorganic $\mathrm{P}(\mathrm{Pi}, \mathrm{mg} \%)$ and $\mathrm{Ca}$ $(m g \%)$ on the experimental time (days, $D$ ) in the treatments

Tratamento Regressão $\quad$ Significância do efeito $\mathrm{r}^{2}$

Treatment Regression Significance of

\begin{tabular}{llcl} 
& \multicolumn{3}{c}{ the effect } \\
\hline 1 & $\mathrm{Pi}=7,78-0,005 \mathrm{D}$ & $* *$ & 0,09 \\
2 & $\mathrm{Pi}=4,87$ & & - \\
3 & $\mathrm{Pi}=4,12-0,009 \mathrm{D}$ & $* * *$ & 0,44 \\
1 & $\mathrm{Ca}=10,22$ & $\mathrm{NS}$ & - \\
2 & $\mathrm{Ca}=11,45$ & $\mathrm{NS}$ & - \\
3 & $\mathrm{Ca}=10,87+0,014 \mathrm{D}$ & $* * *$ & 0,29 \\
\hline
\end{tabular}

Níveis de significância: ${ }^{* *} \mathrm{P}<0,01 ;{ }^{* * *} \mathrm{P}<0,001$.

Levels of significance: ${ }^{* *} P<.01 ;{ }^{\star * *} P<.001$.

O teor de Ca plasmático aumentou com o tempo apenas no tratamento 3 (Tabela $4, \mathrm{P}<0,001$ ). A elevação do Ca plasmático na deficiência de $\mathrm{P}$ é relatada com frequiência na literatura (BREVES et al., 1985; TERNOUTH e SEVILLA, 1990) e pode estar ligada ao aumento da reabsorção óssea e/ou à redução na formação de osso (SCOTT et al., 1997).

A concentração de hidroxiprolina na urina é o mais usado indicador de reabsorção óssea. A hidroxiprolina é um aminoácido típico dos colágenos, sendo encontrada primariamente nos ossos. A hidroxiprolina oriunda da degradação do colágeno não pode ser reutilizada, sendo excretada na urina na forma livre ou ligada a peptídeos (CALVO et al., 1996). A medida da concentração de hidroxiprolina plasmática, devido à facilidade de amostragem, foi preferida à medida na urina, sendo ambas de igual valor diagnóstico (FUJII et al., 1981).

Neste estudo, não foi observada qualquer alteração nos níveis de hidroxiprolina em função do tempo (Tabela 5). Este resultado pode ser atribuído parcialmente à grande variabilidade da medida (coeficiente de variação $=30 \%$ ), também observada por WADSWORTH et al. (1990) e CALVO et al. (1996).

Diversos fatores contribuem para as variações na concentração de hidroxiprolina, entre eles estão a síntese de colágeno (cerca de 1/3 do colágeno recémsintetizado é degradado), a degradação dos demais tecidos conectivos e proteínas séricas e a existência de ritmo circadiano (CALVO et al., 1996). Assim, não é possível excluir totalmente a possibilidade de maior reabsorção óssea nos animais deficientes.

Os animais do tratamento 3 apresentaram aumento acentuado $(\mathrm{P}<0,01)$ da fosfatase alcalina (Tabela 5). Níveis elevados de fosfatase alcalina - uma enzima associada à formação do osso - foram observados em situações em que há distúrbios na mineralização, como na deficiência de P (RADOSTITIS et al., 1994; SCOTT et al., 1997). O tratamento 2 , embora não tenha mostrado variação significativa $(\mathrm{P}>0,05)$ da atividade com o tempo, teve valores médios superiores aos observados no tratamento 1 . O tratamento 1 não apresentou variação com o tempo $(\mathrm{P}>0,05)$, apresentando níveis médios relativamente baixos.

O hematócrito (Tabela 6) apresentou redução significativa, em função do tempo, no tratamento 1 $(\mathrm{P}<0,01)$. Não houve qualquer modificação neste parâmetro nos tratamentos 2 e 3 . O volume de sangue é proporcional ao peso vivo. É possível que animais exibindo maior taxa de crescimento apresentem descompasso parcial entre a síntese de hemáceas e o ganho de massa muscular, levando à redução do hematócrito.

Não houve qualquer efeito do tratamento na temperatura (Tabela 6). A hipertermia (temperatura retal superior a $39,5^{\circ} \mathrm{C}$ ) pode ser provocada por excessiva produção de calor, devido à elevação da atividade metabólica (RADOSTITIS et al., 1994), que pode ser associada à alimentação dos animais (WEBSTER, 1980) poucas horas antes da tomada de temperatura. Os exames clínicos efetuados ao longo do experimento mostraram progressiva queda na condição geral dos animais do tratamento 3: mucosas pálidas, espessamento e aspereza do pêlo, escoliose, magreza e evidência de depravação do apetite (alotriofagia).

Analisando as concentrações de $\mathrm{P}$ nas fezes,

Tabela 5 - Regressão linear relacionando fosfatase alcalina (FA, U/L) e hidroxiprolina (OHPRO, $\mu \mathrm{mol} / \mathrm{L}$ ) no sangue ao tempo de experimentação (dias, D), nos tratamentos

Table 5 - Linear regressions relating alkaline phosphatase $(F A, U / L)$ and hydroxyproline (OHPRO, $\mu \mathrm{mol} / \mathrm{L})$ in blood to experimental time (days, $D$ ) for treatments

\begin{tabular}{lccc}
\hline $\begin{array}{l}\text { Tratamento } \\
\text { Treatment }\end{array}$ & $\begin{array}{c}\text { Regressão } \\
\text { Regression }\end{array}$ & $\begin{array}{c}\text { Significância do efeito } \\
\text { Significance } \\
\text { ofthe effect }\end{array}$ & $\mathrm{r}^{2}$ \\
\hline 1 & $\mathrm{FA}=192,26$ & & - \\
2 & $\mathrm{FA}=309,58$ & $* *$ & - \\
3 & $\mathrm{FA}=267,54+1,113 \mathrm{D}$ & $*, 22$ \\
1 & OHPRO $=48,46$ & & - \\
2 & OHPRO $=48,34$ & & - \\
3 & OHPRO $=54,44$ & & - \\
\hline
\end{tabular}

Níveis de significância: ** $P<0,01$.

Levels of significance: ${ }^{\star *} P<.01$. 
Tabela 6 - Regressão linear relacionando hematócrito (HEM, \%) e temperatura (TEMP, $\left.{ }^{\circ} \mathrm{C}\right)$ tempo de experimentação (dias, D)

Table 6 - Linear regressions relating hematocrite (HEM, \%) and temperature $\left(T E M P,{ }^{\circ} \mathrm{C}\right)$ on experimental time (days, D) for treatments

\begin{tabular}{lccc}
\hline $\begin{array}{l}\text { Tratamento } \\
\text { Treatment }\end{array}$ & $\begin{array}{l}\text { Regressão } \\
\text { Regression }\end{array}$ & $\begin{array}{c}\text { Significânciadoefeito } \\
\text { Significance } \\
\text { ofthe effect }\end{array}$ & $\mathrm{r}^{2}$ \\
\hline 1 & HEM $=40,62-0,023 \mathrm{D}$ & $* *$ & 0,24 \\
2 & $\mathrm{HEM}=38,35$ & & - \\
3 & $\mathrm{HEM}=39,05$ & - \\
1 & $\mathrm{TEMP}=39,65$ & & - \\
2 & TEMP $=39,43$ & & - \\
3 & TEMP $=39,61$ & & - \\
\hline
\end{tabular}

Níveis de significância**, $\mathrm{P}<0,01$.

Levels of significance: ${ }^{*}, P<.01$
Tabela 7 - Regressão linear da concentração de $\mathrm{P}$ na dieta (\%P na matéria seca, Y) em função da concentração fecal do elemento (\%P na matéria seca fecal) obtida por coleta total de fezes (X1) ou amostras retais $(\mathrm{X} 2)$

Table 7 - $\quad$ Linear regressions of $P$ concentration in the diet $(\% P$ in the dry matter, $Y$ ) on the $P$ concentration in the feces (\%P in feces dry matter) obtained by total collection (X1) or using grab samples (X2)

\begin{tabular}{lcc}
\hline $\begin{array}{c}\text { Regressão } \\
\text { Regression }\end{array}$ & $\begin{array}{c}\text { Significância do efeito } \\
\text { Significance } \\
\text { of the effect }\end{array}$ & $\mathrm{r}^{2}$ \\
\hline $\mathrm{Y}=0,019+0,3637 \mathrm{X} 1$ & $* *$ & 0,28 \\
$\mathrm{Y}=-0,012+0,3644 \mathrm{X} 2$ & $* *$ & 0,22 \\
\hline
\end{tabular}

Níveis de significância: **, $\mathrm{P}<0,01$.

Level of significance: ${ }^{* *}, P<.01$

Tabela 8 - Regressão linear relacionando concentrações fecais de P (\%P) na matéria seca (YMS) ou na matéria orgânica (YMO), obtidas de amostras retais, à quantidade do elemento na dieta $(\mathrm{g} / \mathrm{dia}, \mathrm{X})$

Table 8 - $\quad$ Linear regressions relating $P$ fecal concentration $(\% P)$ in the dry matter $(Y M S)$ or in the organic matter (YMO), obtained from grab samples, on the amount of $P$ in the diet ( $g /$ day, $X)$

\begin{tabular}{|c|c|c|c|}
\hline $\begin{array}{l}\text { Dias de experimentação } \\
\text { Days in the trial }\end{array}$ & $\begin{array}{l}\text { Regressão } \\
\text { Regression }\end{array}$ & $\begin{array}{l}\text { Significância do efeito } \\
\text { Significance of the effect }\end{array}$ & $\mathrm{r}^{2}$ \\
\hline 12 & $\mathrm{YMS}=0,4055$ & & - \\
\hline 26 & $\mathrm{YMS}=0,5872$ & & - \\
\hline 151 & $\mathrm{YMS}=0,3808$ & & - \\
\hline 186 & $\mathrm{YMS}=0,3412+0,0145 \mathrm{X}$ & $*$ & 0,33 \\
\hline 192 & $\mathrm{YMS}=0,4065$ & & - \\
\hline 224 & $\mathrm{YMS}=0,4318$ & & - \\
\hline 250 & $\mathrm{YMS}=0,2661+0,0190 \mathrm{X}$ & $*$ & 0,37 \\
\hline 12 & $\mathrm{YMO}=0,5414$ & NS & - \\
\hline 26 & $\mathrm{YMO}=0,7145$ & NS & - \\
\hline 151 & $\mathrm{YMO}=0,7936$ & NS & - \\
\hline 186 & $\mathrm{YMO}=0,4328$ & NS & - \\
\hline 192 & $\mathrm{YMO}=0,5510$ & NS & - \\
\hline 224 & $\mathrm{YMO}=0,6011$ & NS & - \\
\hline 250 & $\mathrm{YMO}=0,4591+0,0224 \mathrm{X}$ & $*$ & 0,37 \\
\hline
\end{tabular}

Níveis de significância: ${ }^{*} P<0,05$.

Levels of significance: ${ }^{*} P<.05$. 
nota-se que as equações de regressão encontradas (Tabela 7) foram muito semelhantes àquelas descritas por HOLECHECK et al. (1985) e MOIR (1960), para a estimativa da concentração de $\mathrm{P}$ na dieta, a partir da concentração de $\mathrm{P}$ na matéria seca orgânica fecal. Aparentemente, o fato de serem utilizadas amostras de fezes coletadas diretamente do reto não alterou a equação obtida com a coleta total de fezes.

Existe, contudo, importante limitação à expressão de $\mathrm{P}$ nas fezes em porcentagem, pois, como a redução no consumo de alimento é provavelmente o fator isolado mais importante na deficiência de P (TERNOUTH e SEVILLA, 1990), pode haver elevação na concentração de $\mathrm{P}$ fecal na massa excretada, comprometendo o valor diagnóstico desta medida. Observações neste sentido foram relatadas por READ et al. (1986) e WADSWORTH et al. (1990). Os dados da Tabela 8 reforçam estes achados, em que a porcentagem de $\mathrm{P}$ na matéria seca ou na matéria orgânica fecal não apresentou variação consistente $(\mathrm{P}>0,05)$, em função do nível de $\mathrm{P}$ na dieta (g/dia), exceto aos 186 dias na matéria seca e aos 250 dias de experimento na matéria seca e matéria orgânica. Cabe lembrar que a queda no desempenho foi evidenciada aos 71 dias. Assim, concluiu-se que seria mais apropriada a determinação de excreção fecal total de P (g/dia), como sugerido por READ et al. (1986), o que tornaria a utilização deste parâmetro pouco viável para animais a campo.

\section{Conclusões}

Os parâmetros ósseos mais sensíveis à deficiência de $\mathrm{P}$ foram a concentração de $\mathrm{P}$ no osso fresco ou expressa em relação ao volume de osso. As concentrações de $\mathrm{P}$ no osso encontradas na literatura e usadas como referência de adequação/deficiência de fósforo não devem ser utilizadas isoladamente. Os novilhos deficientes em $\mathrm{P}$ apresentaram níveis plasmáticos ao redor de $3,0 \mathrm{mg} \%$ na época em que houve redução do apetite e conseqüente perda de peso. Estas concentrações foram mantidas até o final do experimento. Observou-se também aumento da atividade da fosfatase alcalina total e do cálcio no plasma na deficiência de $\mathrm{P}$, indicando alterações no metabolismo mineral, sem qualquer alteração na concentração de hidroxiprolina plasmática. A concentração de $\mathrm{P}$ em amostras de fezes coletadas diretamente do reto não permitiram a detecção precoce da deficiência de $\mathrm{P}$, embora exista possibilidade de estimativa do P ingerido a partir do $\mathrm{P}$ total (g/dia) excretado nas fezes.

\section{Referências Bibliográficas}

BELONJE, P.C. 1978. An investigation into possible methods of assessing the intake of calcium and phosphorus by grazing sheep. Onderstepoort J. Vet. Res., 45 (1):7-22.

BORTOLUSSI, G., TERNOUTH, J.H., McMENIMAN, N.P. 1996. Dietary nitrogen and phosphorus depletion in cattle and their effects on liveweight gain, blood metabolite concentrations and phosphorus kinetics. J. Agric. Sci., 126(4):493-501.

BREVES, G., ROSS, R., HOELlER, H. 1985. Dietary phosphorus depletion in sheep: effects on plasma inorganic phosphorus, calcium, $1,25-(\mathrm{OH})_{2}$-vit. $\mathrm{D}_{3}$ and alkaline phosphatase and on gastrointestinal $\mathrm{P}$ and $\mathrm{Ca}$ balances. J. Agric. Sci., 105(3):623-629.

BROMMAGE, R., ANTILLE, S., PITTET, S. et al. 1992. Influence of dietary calcium content on bone mass accumulation in growing female rats. J. Bone Min. Res., 7:S271.

CALVO, M.S., EYRE, D.R., GUNDBERG, C.M. 1996. Molecular basis and clinical application of biological markers of bone turnover. Endocr. Rev.,17(4):333-368.

CHALLA, J., BRAITHWAITE, G.D., DHANOA, M.S. 1989. Phosphorus homoeostasis in growing calves. J. Agric. Sci., 112:217-226.

COATES, D.B., TERNOUTH, J.H. 1992. Phosphorus kinetics of cattle grazing tropical pastures and implications for the estimation of their phosphorus requirements. J. Agric. Sci., 119:401-409.

CORLETT, S.C., CARE, A.D. 1988. The effects of reduced dietary phosphate intake on plasma osteocalcin levels in sheep. Q. J. Exp. Phys.,73 (3):443-445.

ECKLES, C.H., GULLICKSON, T.W., PALMER, L.S. 1932. Phosphorus deficiency in the ration of cattle. Tech. Bull. Minnesota Agric. Exp. Sta., (91):1-118.

FICK, K.R., McDOWELL, L.R., MILES, J.H. et al. 1980. Métodos de análises de minerais em tecidos de animais e de plantas. 2.ed. Gainesville: University of Florida. v.1. Paginação descontínua.

FUJII, Y., WATANABE, H., UEDA, Y. et al. 1981. Diagnostic values of hydroxyproline in horse serum. Bull. Eq. Res. Inst., (18):73-83.

GARTNER, R.J., McLEAN, R.W., LITTLE, D.A. et al. 1980. Mineral deficiencies limiting production of ruminants grazing tropical pastures in Australia. Trop. Grassl., 14(3):266-272.

HARRIS, L.E. 1970. Os métodos químicos e biológicos empregados na análise de alimentos. Gainesville: University of Florida. Paginação descontínua.

HOEY, W.A., MURPHY, G.M., GARTNER, R.J.W. 1982. Whole body composition of heifers in relation to phosphorus status with particular reference to skeleton. J. Agric. Sci., 98(1):31-37.

HOLECHECK, J.L., GALYEAN, M.L., WALLACE, J.D. et al. 1985. Evaluation of faecal indices for predicting phosphorus status of cattle. Grass Forage Sci., 40(4):489-492.

JAESCKE, G. 1975 Fur routinemaebigen Bestimmung des frien hydrocyprolins in pferdeserum. Zentralbl. Veterinaermed. A, 22(2):89-101.

LAWRENCE, T.L., FOWLER, V.R. 1997. Growth of farm animals. Wellington: CAB International. 330p.

LEGROS, R., BALMAIN, N., BONEL, G. 1987. Age-related changes in mineral of rat and bovine cortical bone. Calcif. Tiss. Int., 41(3):137-144.

LITTLE, D.A. 1984. Definition of an objective criterion of body phosphorus reserves in cattle and its evaluation "in vivo". Can. J. Anim. Sci., 64(Suppl.):229-231. 
LITTLE, D.A. 1972. Bone biopsy in cattle and sheep for studies of phosphorus status. Aust. Vet. J., 48(12):668-670.

LITTLE, D.A., McLEAN, R.W., WINTER, W.M. 1977. Prediction of the phosphorus content of herbage consumed by grazing cattle. J. Agric. Sci., 88(31):533-538.

LITTLE, D.A., SHAW, N.H. 1979. Superphosphate and stocking rate effects on a native pasture oversown with Sthylosantehes humilis in central coastal Queensland. 5. Bone phosphorus levels in grazing cattle. Aust. J. Exp. Agric. Anim. Husb., 19(101):645-651.

LITTLE, D.A., McMENIMAN, N.P. 1975. Variation in bone composition of grazing sheep in south-western Queensland, related to lactation and type of country. Aust. J. Exp. Agric. Anim. Husb., 13(62):229-233.

McDOWELL, L.R. 1985. Detection of mineral status of grazing ruminants. In: CUNHA, T.J.(Ed.) Nutrition of grazing ruminants in warm climates. Orlando: Academic Press. p.339-357.

MELLO JR., F.C., FIELD, R.A., RILEY, M.L. 1978. Effect of age and anatomical location on composition of bovine bone. J. Food Sci., 43(3):677-679.

MOIR, K.W. 1960. Nutrition of grazing cattle. 2. Estimation of phosphorus and calcium in pastures selected by grazing cattle. Queensl. J. Agric. Sci., 17(4):373-383.

NICODEMO, M.L.F. Measurement and function of turnover markers in sheep and pig bone. Aberdeen, Escócia: Universidade de Aberdeen, 1997. 203p. Tese (Doutorado em Agricultura) - Faculdade de Agricultura/Universidade de Aberdeen, 1997.

RADOSTITIS, O.M., BLOOD, D.C., GAY, C.C. 1994. Veterinary Medicine. 8.ed. London: Bailliere Tindall.

READ, M.V.P., ENGELS, E.A.N. 1986. Phosphorus and the grazing ruminant. 3. Rib bone samples as an indicator of the P status of cattle. S. Afr. J. Anim. Sci., 16(1):13-17.

RESOURCE CONSULTING SERVICES (Darwin, Australia).1986. A review of phosphorus requirements of grazing cattle in North Australia. Darwin. 12p.

SAS Institute (Cary, USA). 1985. Statistical analysis system user's guide: basics. Cary. 1290p.

SCOTT, D., LOVERIDGE, N., NICODEMO, L. et al. 1997. Effect of diets varying in nitrogen or phosphorus content on indicators of bone growth in lambs. Exp. Phys., 82(1):193-202.

SCOTT, D., ROBINS, P., NICOL, P. et al. 1994. Effects of low phosphate intake on bone and mineral metabolism and microbial protein synthesis in lambs. Exp. Phys., 79(2):183-187.

SCOTT, D., WHITELAW, F.G., BUCHAN, W. et al. 1985. The effect of variation in phosphorus intake on salivary phosphorus secretion, net intestinal phosphorus absorption and faecal endogenous excretion in sheep. J. Agric. Sci., 105(2):271-277.
SHIRAZI-BEECHEY, A.P., BEECHEY, R.B., PENNY, J. et al. 1991. Mechanisms of phosphate transport in sheep intestine and parotid gland: response to variation in dietary phosphate supply. Exp. Phys., 76(2):231-241.

SHUPE, J.L., BUTCHER, J.E., CALL, J.W. et al. 1988. Clinical signs and bone changes associated with phosphorus deficiency in beef cattle. Am. J. Vet. Res., 49(9):1629-1636.

TERNOUTH, J.H., McLACHLAN, B.P., CLARKE, J.M. et al.1993. Effects of dietary phosphorus and nitrogen deficiencies on the intake, growth and metabolism of lambs. J. Agric. Sci., 121:409-419.

TERNOUTH, J.H., SEVILLA, C.C. 1990. The effects of low levels of dietary phosphorus upon the dry matter intake and metabolism of lambs. Aust. J. Agric. Res. 41(1):175-184.

UNDERWOOD, E.J. 1981. The mineral nutrition of livestock. 2.ed. Farnham Royal: Commonwealth Agricultural Bureaux. $180 \mathrm{p}$.

UNDERWOOD, E.J. 1966. The mineral nutrition of livestock. Aberdeen: FAO/CAB. 237p.

WADSWORTH, J.C., McLEAN, R.W., COATES, D.B. et al. 1990. Phosphorus and beef production in northern Australia. 5. Animal phosphorus status and diagnosis. Trop. Grassl., 24(3):185-196.

WAN ZAHARI, M., THOMPSON, J.K., SCOTT, D. et al. 1990. The dietary requirements of calcium and phosphorus for growing lambs. Anim. Prod., 50(2):301-307.

WEBSTER, A.J.F. 1980. Energy costs of digestion and metabolism in the gut. In: RUCKEBUSCH, Y., THIVEND, P. (Eds.) Digestive physiology and metabolism in ruminants. Westport: AVI. v.2. p. 469-484.

WINTER, W.H. 1988. Supplementation of steers grazing Stylosanthes hamata pastures of Kathrine, Northern Territory. Aust. J. Exp. Agric., 28(6):669-682.

WISE, M.B., SMITH, S.E., BARNER, L.L. 1958. The phosphorus requirements of calves. J. Anim. Sci., 17(1):89.

Recebido em: 24/06/98

Aceito em: 13/10/99 